\title{
Reference ranges for the fetal mesencephalon to occiput measurement at 11 to $13+6$ weeks of gestation
}

\author{
DRAGOS NEMESCU ${ }^{1,2}$, ANA MARIA ADAM ${ }^{1}$, INGRID ANDRADA TANASA ${ }^{1}$, DEMETRA SOCOLOV ${ }^{1}$, \\ ROXANA ELENA BOHILTEA ${ }^{2,3}$, DAN BOGDAN NAVOLAN ${ }^{3,4}$ and MONA ELENA ZVANCA ${ }^{2,3}$ \\ ${ }^{1}$ Department of Obstetrics and Gynecology, 'Grigore T. Popa' University of Medicine and Pharmacy, 700115 Iasi; \\ ${ }^{2}$ 'Euromedicenter' Medical Centre, 700469 Iasi; ${ }^{3}$ Department of Obstetrics and Gynecology, 'Carol Davila' University \\ of Medicine and Pharmacy, 020021 Bucharest; ${ }^{4}$ Department of Obstetrics and Gynecology, \\ ‘Victor Babes' University of Medicine and Pharmacy, 300041 Timisoara, Romania
}

Received April 10, 2020; Accepted May 13, 2020

DOI: $10.3892 /$ etm.2020.8803

\begin{abstract}
The objective was to have a quantitative description of the normal position of the fetal midbrain in the first trimester, through defining the reference ranges for the mesencephalon to the occipital bone distance, in the axial plane. This was a prospective study that included normal fetuses screened between 11 and 13 weeks of gestation. The distance was measured between the posterior limit of the mesencephalon to the occipital bone in the same axial view as the one required for the biparietal diameter (BPD) assessment, at this gestational age (GA). The reference ranges using quantile regression, according to the crown-rump length (CRL), BPD, and GA were fitted. Data analysis included 428 ultrasound measurements. A good, linear correlation was observed between mesencephalon to occiput (MO) distance and CRL, BPD, or GA. It increased linearly with advancing gestation $\left(\log _{10} \mathrm{MO}=-0.1834+0.0092 \times \mathrm{CRL}, \mathrm{R}^{2}=0.48, \mathrm{P}<0.0001\right)$ and was independent of maternal demographic characteristics and intracranial translucency (IT). In our study, the 1st percentile of the normal MO distance varies from $1.31 \mathrm{~mm}$ at a CRL of $45 \mathrm{~mm}$ to $2.08 \mathrm{~mm}$ at a CRL of $84 \mathrm{~mm}$. The intraclass correlation coefficient (ICC) was 0.89 for intraobserver variability. A significant increase in the MO distance was found in the patients who did not receive folic acid in the first trimester
\end{abstract}

Correspondence to: Dr Roxana Elena Bohiltea, Department of Obstetrics and Gynecology, 'Carol Davila' University of Medicine and Pharmacy, Splaiul Independenței 169, 020021 Bucharest, Romania

E-mail: r.bohiltea@yahoo.com

Dr Ana Maria Adam, Department of Obstetrics and Gynecology, 'Grigore T. Popa' University of Medicine and Pharmacy, 16 Universitatii Street, 700115 Iasi, Romania

E-mail: adam.anamaria89@gmail.com

Key words: first-trimester ultrasound, mesencephalon, midbrain, prenatal diagnosis, spina bifida of pregnancy [1.056 vs. 1.008 multiple of median (MoM), $\mathrm{P}=0.014]$. A simple measurement is described between the midbrain and the occipital bone, obtained in the same axial view. It increases linearly with advancing gestation. Integration of this measurement into the routine ultrasound screening in association with the 'crash sign' and recognizing the lower extreme values could lead to an early diagnosis of open spina bifida (OSB).

\section{Introduction}

Open spina bifida (OSB) is a severe congenital anomaly of the central nervous system associated with a wide range of neurological handicaps (1). In the last 10 years, there is an increasing interest in diagnosing OSB at 11-13 weeks of gestation. This interest has been promoted by advances of the ultrasound equipment and progressive understanding of the abnormal arrangement of the posterior fossa because of the continuous leakage of cerebrospinal fluid (CSF) $(2,3)$. The local pressure gradient leads to caudal displacement of the brainstem (BS) with the obliteration of the cisterna magna $(4,5)$.

Consequently, different secondary markers have been described to improve the early detection of spina bifida. They reflect specific characteristics of this displacement but have yet to be introduced into practice. In the mid-sagittal view of the head, the most extensively researched markers for OSB were intracranial translucency (IT) (6-8), BS diameter, brainstem to occipital bone (BSOB) distance, $\mathrm{BS} / \mathrm{BSOB}$ ratio and the junction between the midbrain and the BS below the maxilla-to-occipital line $(5,9,10)$. OSB markers in the axial view of the head are less studied: ratio of choroid plexus size to head size (3), biparietal diameter (BPD) (11), posterior displacement of the midbrain $(4,12)$ and the distance between the occipital bone and the aqueduct of Sylvius (AoS) $(12,13)$.

Recently there is an increasing interest in the evaluation of the fetal head in the axial view through description of simple signs, which can be easily recognized at the time of BPD measurement: the dry brain and the crash sign $(3,4)$. However, the posterior displacement of the midbrain and its impact with the occipital bone could vary and may not be present in all cases in the first trimester (4). Therefore, we found that there is 
a need for a quantitative description of the normal position of the fetal midbrain. This study aimed to describe the reference ranges for the distance from the mesencephalon to the occipital bone in our population, in the axial plane, at 11 to $13+6$ weeks of gestation.

\section{Patients and methods}

This is a prospective study that included women attending for aneuploidy risk assessment at 11-13 gestational weeks, between 2018 and 2019, at a prenatal diagnostic center (private practice). It was part of a protocol approved by the Institutional Board of the 'Euromedicenter' Medical Centre (Iasi, Romania) and all patients provided signed informed consent for fetal examination. The patients were informed about the limitations of the first-trimester ultrasound in detecting anomalies and were invited for a second-trimester fetal anomaly scan. All viable, singleton pregnancies with a crown-rump length (CRL) of 45-84 $\mathrm{mm}$ and without fetal structural or chromosomal abnormalities were included. The examinations were performed by experienced sonographers, accredited by Fetal Medicine Foundation (FMF) for first-trimester screening. The scans were started transabdominally, and if the visualization of the markers was inadequate, a transvaginal ultrasound was offered. Ultrasound examinations were performed with Voluson E10 and E8 machines, equipped with transabdominal RM-6C and RAB4-8D transducers (GE Medical Systems).

The GA was checked according to the CRL. Baseline maternal characteristics and ultrasound data, such as nuchal translucency (NT) measurement and presence of fetal anomalies, were recorded within the Astraia database (Astraia Software Gmbh) as a part of routine clinical practice.

On the mid-sagittal view of the fetal profile, the IT was also assessed, using the method described by Chaoui et al (6). BPD was measured on an axial view of the fetal cranium, with a symmetrical section of the brain, that included the midline echo and choroid plexuses, the calipers placed outer-to-inner borders of the skull (14).

The distance between the posterior limit of the mesencephalon and the occipital bone was measured in an axial view of the head, as recommended by the International Society of Ultrasound in Obstetrics and Gynecology (ISUOG) for BPD measurements at this gestational age (GA) (15). This plane, also described by Finn et al (12), was acquired through a cranial sweep in the axial plane, excluding almost all the choroid plexus in the lateral ventricles. Here, 'the mesencephalon is visualized as a semicircular structure in the posterior brain and appears as a continuation of the thalami', presenting the cerebral aqueduct of Sylvius (AoS) centrally (Ushakov et al) (4). The image was magnified, so as the fetal head occupy almost the whole screen. The calipers were placed on the posterior border of the mesencephalon and the anterior border of the occiput, on the lines that define the borders, using the same technique as for NT measurement. The bony occiput was carefully differentiated from the occipital cortex (Fig. 1).

The images were retrospectively assessed, and only high-quality measurements were included for subsequent analysis and development of reference ranges. When more than two images were available, we selected two of the highest quality.

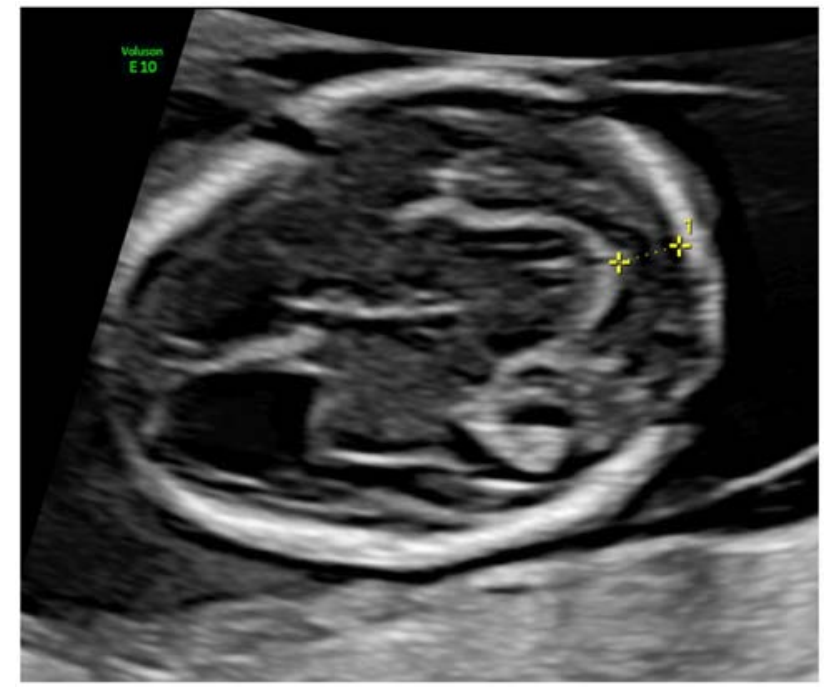

Figure 1. Standard transabdominal axial view at the level of thalami and the aqueduct of Sylvius (AoS), demonstrating the measurement of the mesencephalon to occipital bone distance.

All patients had a normal spine and brain structures scan in the second trimester according to the ISUOG guidelines (15), and pregnancy outcomes were retrieved from the hospital database and by direct questioning of the parents.

Statistical analysis. Continuous variables were reported as medians and interquartile ranges, while categorical variables as numbers and percentages. The normality of data was assessed using the Kolmogorov-Smirnov test. The distribution of the measurements was made close to Gaussian through logarithmic transformation. The maternal weight and maternal body mass index (BMI) distributions were made Gaussian after reciprocal transformation. The multiple of median (MoM) values for IT and mesencephalon to occiput (MO) measurements were calculated using the expected values from the regression equations described by Kappou et al (16) and our developed formula, respectively. Finally, the MoMs distributions were made Gaussian trough logarithmic transformation. The relationship between MO distance MoM and other maternal and fetal parameters was investigated using Pearson's correlation coefficient. Continuous variables were compared using Student's t-test. The interobserver variability was evaluated using the intraclass correlation coefficient (ICC).

The mean and standard deviation polynomial models were evaluated for the measured data using the least-squares regression. Regression coefficients for the standard deviation were obtained by regressing the scaled standardized absolute residuals on studied independent variables (13). We identified and excluded from the initial model significant outliers with standardized residuals. The normal distribution of the residuals and model premises was verified through histograms, probability plots, and quantile-quantile (Q-Q) plots of standardized residuals. Quantile regression was used to compute the different percentiles of the studied measurements.

Statistical analysis was performed with the SPSS 25.0 software (SPSS Inc.) and R software with quantreg and ggplot2 packages for quantile regression and graphical representation 
Table I. Regression coefficients for the mean and standard deviation of the logarithm of mesencephalon to occipital bone distance $\left(\log _{10} \mathrm{MO}\right)$ with respect to CRL, BPD and GA in days.

\begin{tabular}{|c|c|c|c|c|c|}
\hline \multirow[b]{2}{*}{ Independent variable } & \multirow[b]{2}{*}{$\mathrm{R}^{2}$} & \multicolumn{2}{|c|}{ Mean } & \multicolumn{2}{|c|}{ Standard deviation } \\
\hline & & Intercept & Coefficient & Intercept & Coefficient \\
\hline CRL & 0.479 & -0.1833513 & 0.0092027 & 0.568432 & 0.003407 \\
\hline BPD & 0.507 & -0.30942 & 0.03309 & 0.866793 & -0.003963 \\
\hline GA & 0.421 & -1.0494882 & 0.0164975 & 0.7289963 & 0.0006472 \\
\hline
\end{tabular}

$\mathrm{R}^{2}$, adjusted $\mathrm{R}$ squared of the models. CRL, crown-rump length in millimeters; BPD, biparietal diameter in millimeters; GA, gestational age.

Table II. Percentiles (1st, 5th, 50th, 95th, and 99th) for mesencephalon to occipital bone distance, with respect to CRL, BPD and GA, derived through quantile regression in a population of 385 fetuses without spina bifida.

\begin{tabular}{|c|c|c|c|c|c|}
\hline \multirow[b]{2}{*}{ Variable } & \multicolumn{5}{|c|}{ Percentiles } \\
\hline & $1 \mathrm{st}$ & 5 th & 50 th & 95 th & 99th \\
\hline \multicolumn{6}{|c|}{ CRL (mm) } \\
\hline 45 & 1.31 & 1.36 & 1.7 & 2.3 & 3.22 \\
\hline 50 & 1.43 & 1.45 & 1.89 & 2.53 & 3.45 \\
\hline 55 & 1.51 & 1.61 & 2.11 & 2.79 & 3.7 \\
\hline 60 & 1.6 & 1.79 & 2.34 & 3.08 & 3.96 \\
\hline 65 & 1.69 & 1.98 & 2.61 & 3.39 & 4.25 \\
\hline 70 & 1.78 & 2.2 & 2.9 & 3.74 & 4.55 \\
\hline 75 & 1.88 & 2.43 & 3.23 & 4.12 & 4.88 \\
\hline 80 & 1.99 & 2.7 & 3.59 & 4.54 & 5.22 \\
\hline 84 & 2.08 & 2.93 & 3.91 & 4.91 & 5.52 \\
\hline \multicolumn{6}{|c|}{$\mathrm{BPD}(\mathrm{mm})$} \\
\hline 16 & 1.22 & 1.26 & 1.62 & 2.2 & 2.9 \\
\hline 18 & 1.42 & 1.43 & 1.9 & 2.56 & 3.29 \\
\hline 20 & 1.59 & 1.67 & 2.22 & 2.97 & 3.73 \\
\hline 22 & 1.78 & 1.95 & 2.6 & 3.45 & 4.23 \\
\hline 24 & 2 & 2.27 & 3.05 & 4.01 & 4.8 \\
\hline 26 & 2.24 & 2.65 & 3.57 & 4.66 & 5.45 \\
\hline 28 & 2.52 & 3.1 & 4.18 & 5.41 & 6.18 \\
\hline 30 & 2.83 & 3.62 & 4.89 & 6.28 & 7.01 \\
\hline \multicolumn{6}{|c|}{ GA (days) } \\
\hline 77 & 1.29 & 1.31 & 1.61 & 2.23 & 3.17 \\
\hline 80 & 1.4 & 1.43 & 1.82 & 2.49 & 3.42 \\
\hline 83 & 1.5 & 1.59 & 2.05 & 2.79 & 3.7 \\
\hline 86 & 1.6 & 1.76 & 2.31 & 3.12 & 4 \\
\hline 89 & 1.72 & 1.96 & 2.6 & 3.49 & 4.32 \\
\hline 92 & 1.84 & 2.17 & 2.93 & 3.9 & 4.67 \\
\hline 95 & 1.97 & 2.42 & 3.3 & 4.36 & 5.05 \\
\hline 98 & 2.11 & 2.68 & 3.72 & 4.88 & 5.46 \\
\hline
\end{tabular}

CRL, crown-rump length; BPD, biparietal diameter; GA, gestational age.

of the data. A P-value of $<0.05$ was considered statistically significant.

\section{Results}

In total, 385 pregnant women were included in our study, for whom we performed 428 measurements of the mesencephalon to occipital distance. The median maternal age was 32 years (interquartile 29-35), and 100\% of the women were Caucasians. Maternal weight had a median of $62 \mathrm{~kg}$ (interquartile 56-69), and the median BMI was $22.7 \mathrm{~kg} / \mathrm{m}^{2}$ (interquartile 20.7-25.8). The median GA at examination was 12.7 weeks (interquartile 12.3-13.1). At the same time, CRL and BPD had a median of $65 \mathrm{~mm}$ (interquartile 60-71) and $22 \mathrm{~mm}$ (interquartile 20-24), respectively. The NT had a median of $1.8 \mathrm{~mm}$ (interquartile 1.5-2.1), and that for IT was $1.8 \mathrm{~mm}$ (interquartile 1.6-2). There were $188(55 \%)$ of nullipara and $27(7.9 \%)$ smokers in the study group. The conception was spontaneous in $306(89.5 \%)$ of the patients, the rest had received in vitro fertilization (24 cases, $7 \%$ ), or ovulation induction (11 cases, 3.2\%), 278 (83.5\%) of patients received folic acid throughout the first trimester of gestation. In $5.8 \%$ of the examinations, the measurements were performed on axial views obtained through a transvaginal ultrasound.

A good, positive correlation was observed between the MO distance and BPD, CRL or GA (Pearson's correlation coefficient of $0.719,0.701$ and $0.654, \mathrm{P}<0.01$ for all). A simple polynomial regression can best describe the relationship between the mean of MO distance and these parameters. Standard deviation also had a linear correlation with BPD, CRL, or GA. The regression coefficients of the models are presented in Table I. The scatter plots with the median and the 5th and 95th percentiles are presented in Fig. 2. Table II shows the 1st, 5th, 50th, 95th and 99th percentiles for the fetal MO measurements, according to CRL, BPD, and GA in the first trimester.

We found a significant increase in the MO MoM in the patients who did not receive folic acid in the first trimester of pregnancy [1.056 vs. $1.008 \mathrm{MoM}, \mathrm{t}(115)=-2.49, \mathrm{P}=0.014]$. There were no significant associations between $\mathrm{MO}$ distance MoM, and maternal demographic characteristics (age, parity, weight, BMI, smoking status, mode of conception, MoM IT). The Pearson's correlation coefficients (cc) were, respectively: age ( $c c=0.01, \mathrm{P}=0.43)$, maternal weight $(\mathrm{c}=0.01, \mathrm{P}=0.49)$, maternal BMI $(\mathrm{cc}=0.03, \mathrm{P}=0.31)$ and $\mathrm{IT} \mathrm{MoM}(\mathrm{cc}=0.1, \mathrm{P}=0.04)$. MO measurements MoMs were not significantly different between parous versus nulliparous $(\mathrm{P}=0.25)$, smokers versus 
A

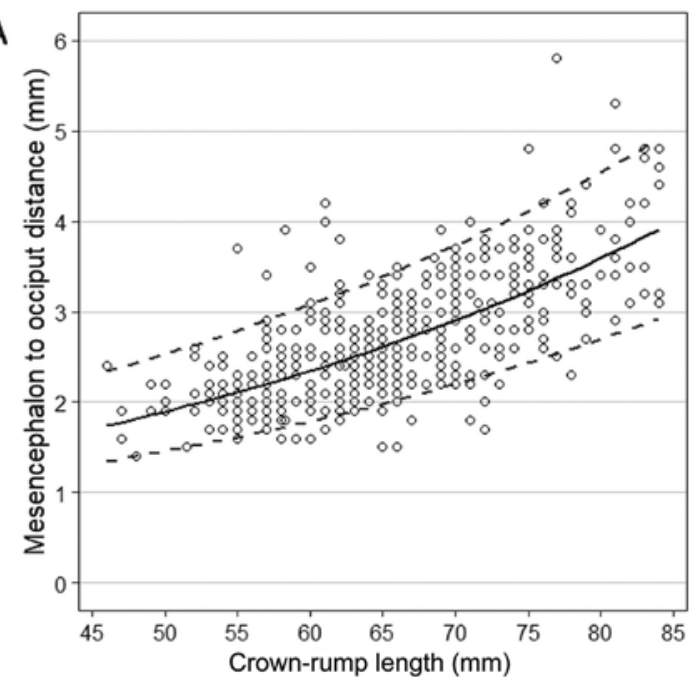

$\mathrm{B}$

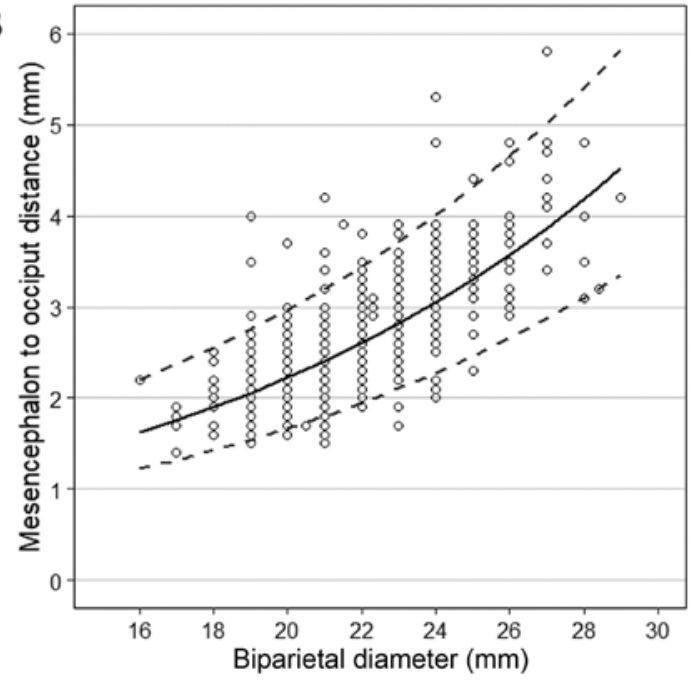

C

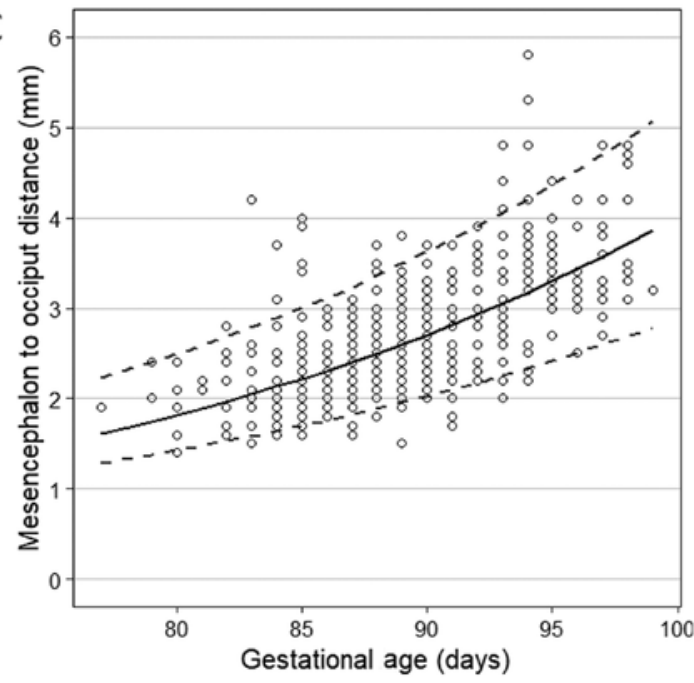

Figure 2. Scatter plots of mesencephalon to occiput measurements with medians (solid lines), the 5th and 95th percentiles (dashed lines) as a function of (A) crown-rump length, (B) biparietal diameter and (C) gestational age.

non-smokers $(\mathrm{P}=0.56)$, and spontaneously conceived pregnancies versus pregnancies resulted from assisted reproduction $(\mathrm{P}=0.24)$.
The intraobserver reproducibility for MO measurement, assessed in a separate group of 42 fetuses, was good, with an ICC of 0.89 (95\% confidence interval 0.81-0.94, $\mathrm{P}<0.001$ ).

\section{Discussion}

Main findings. We measured the MO distance prospectively in the axial view and constructed its reference ranges at 11 to $13+6$ weeks of gestation. These measurements were not significantly associated with the maternal demographic characteristics (age, weight, BMI, parity, smoking status, mode of conception), nor IT. We found a significant increase in the MO distance in the patients who did not receive folic acid in the first trimester of pregnancy. To the best of our knowledge, there are no other studies in the literature evaluating this type of measurement.

Comparison with previous studies. The posterior fossa was evaluated at 11 to $13+6$ weeks, mainly by transabdominal ultrasound, in the midsagittal view of the fetal head. This was linked with the measurement of the indirect signs for OSB, such as IT, BS, and BSOB distance $(5-7,9)$. The reasons behind this were multiple (7). First, this view was used in early screening for aneuploidies; therefore, it was considered that the posterior brain region could be viewed easier, more successfully, in the same mid-sagittal plane. Second, experts believed that an axial view of the posterior fossa is more challenging to achieve by transabdominal ultrasound and needs a transvaginal approach, which cannot be considered as a routine tool for screening. Third, experts thought that the transvaginal ultrasound reveals many details and needs some expertise to understand.

Few studies have evaluated the indirect OSB markers in the axial plane. They mainly described the ultrasound aspects of the posterior displacement of the mesencephalon and its impact with the occipital bone, named recently as a "crash sign' $(2,4,12,13,17)$. Only two studies reported an objective assessment of the midbrain shifting, measuring the distance between the AoS to the occiput $(12,13)$. This aspect could be significant in OSB detection because up to $9 \%$ of fetuses with OSB could have a negative 'crash sign' (4).

In our study, the MO measurement was easily obtainable in an axial plane and did not require extra scanning time, as the required view is the same to the one needed for the BPD measurement (Fig. 1), at this GA (15). We believe that the axial view is less demanding compared with the sagittal one, and it can be especially useful when the fetus has a dorsal-anterior or lateral position. The border of the mesencephalon is more clearly defined, compared with the measurement of the AoS to occiput distance, especially at low GAs.

Moreover, in our prospective study, the need for a transvaginal measurement was significantly lower compared with that found by two retrospective studies that evaluated the AoS to occiput distance (5.8 vs. $30 \%)(12,13)$.

The MO distance, like the AoS-to-occiput distance, had a good correlation with CRL and GA $(12,13)$. Furthermore, the relationship between the means of the markers and the CRL was also linear, which supports the argument that posterior fossa measurements in the axial plane are reproducible and could have clinical use. Like other indirect markers for OSB measured in the sagittal plane (IT or cisterna magna) (18), 
the MO distance does not correlate with maternal demographic characteristics. Also, we found a good, comparable, intraobserver variability with other studies that evaluated the posterior fossa (12).

Strengths and limitations. Our study described a new measurement in the posterior fossa, accounting for its variation in pregnancy by developing reference ranges in healthy fetuses. The regression models provided a good fit, and we can use them in the assessment of future pregnancies. The main advantage of our study is its prospective nature, the measurements being performed during the patient examination. However, our study failed to include measurements from cases with OSB; therefore, we cannot evaluate the efficacity of MO assessment.

Interestingly, in those patients who did not receive folic acid during the first trimester of pregnancy, we found a significant rise in the MO distance. This increase has not been reported previously, it could appear due to increased production of cerebrospinal fluid, and its significance needs to be confirmed by future studies.

Clinical implications. At 11-13 weeks, the fetuses with OSB shows a reduction of the cerebrospinal fluid, due to its leakage at the level of the spinal defect (3). Consequently, others have proposed that there is a reduction of pressure in the spinal cord, cisterna magna, and fourth ventricle, compared with choroid ventricles, which produces a posterior and caudal displacement of the mesencephalon $(4,8)$. We can evaluate this, in an axial view, as an impact and deformation of the mesencephalon on the occipital bone. Ushakov et al (4) described this as a "crash sign', a simple qualitative marker for diagnosing spina bifida. However, up to $9 \%$ of fetuses with confirmed OSB did not have such a definite sign. Therefore, an objective assessment of the distance from the mesencephalon and the occiput is necessary here, to improve the diagnosis. This measurement has the advantage of being performed in the same axial thalamic plane as the one needed for the BPD measurement (Fig. 1). Measurement of BPD is a good practice recommendation at this GA, according to the ISUOG guidelines (15).

In our study, the first percentile of the computed normal range varies from $1.31 \mathrm{~mm}$ at a CRL of $45 \mathrm{~mm}$ to $2.08 \mathrm{~mm}$ at a CRL of $84 \mathrm{~mm}$. There are also available MO percentiles for BPD and GA. These should not be used in practice as a diagnosis of OSB, but as a guide from which one should consider the evaluation for the possibility of a neural tube defect. Recognizing a displaced mesencephalon could alert the operator to the need for a supplementary evaluation. Therefore, a search for other indirect markers in axial and sagittal planes and direct visualization of the spine in axial view by an expert, using a higher resolution with transvaginal probe and 3D volume reconstruction is advised (7,19). Also, the patient could be asked to have a review after two weeks to confirm the diagnosis (12).

In our opinion, the evaluation of the MO distance could be easily integrated into the routine first-trimester screening scan at 11 to $13+6$ gestational weeks. Implementation of this measurement into the routine first-trimester practice could lead to an early diagnosis of OSB, which gives the parents time for an informed decision. They could have the option for a fetal surgery with the intra-uterine closure of the spinal defect, with promising results, but available only in specialized centers (20). Otherwise, early termination of pregnancy is safer and less traumatic for the affected parents.

In conclusion, we described a simple measurement between the mesencephalon and the occipital bone, obtained in the same axial view as the one required for the BPD measurement, in the first-trimester screening. Its reference values have a good correlation with CRL, BPD, and GA. Integration into the routine ultrasound screening in association with the 'crash sign' and recognizing the lower extreme values could lead to an early diagnosis of OSB. Also, the distance seems to increase in those patients who did not received folic acid during the first trimester of pregnancy. We hope that future studies will specify the role of this measurement in early ultrasound.

\section{Acknowledgements}

Not applicable.

\section{Funding}

No funding was received.

\section{Availability of data and materials}

The datasets used and/or analyzed during the current study are available from the corresponding author on reasonable request.

\section{Authors' contributions}

DN and MEZ designed the study. DN performed the measurements within the study. AMA, IAT and DS acquired and assembled the data. DN, REB, DBN and MEZ analyzed and interpreted the data. DN, AMA, IAT, DS, REB, DBN and MEZ were involved in drafting the manuscript and revising it critically for important intellectual content. All authors read and approved the final version of the manuscript.

\section{Ethics approval and consent to participate}

The present study was conducted in accordance with the World Medical Association Declaration of Helsinki and was approved by the Institutional Board of the 'Euromedicenter' Medical Centre (Iasi, Romania). The pregnant women included in the study provided written informed consents.

\section{Patient consent for publication}

Not applicable.

\section{Competing interests}

The authors declare that there are no competing interests.

\section{References}

1. Avagliano L, Massa V, George TM, Qureshy S, Bulfamante GP and Finnell RH: Overview on neural tube defects: From development to physical characteristics. Birth Defects Res 111: 1455-1467, 2019. 
2. Loureiro T, Ushakov F, Montenegro N, Gielchinsky Y and Nicolaides KH: Cerebral ventricular system in fetuses with open spina bifida at 11-13 weeks' gestation. Ultrasound Obstet Gynecol 39: 620-624, 2012.

3. Chaoui R, Benoit B, Entezami M, Frenzel W, Heling KS, Ladendorf B, Pietzsch V, Sarut Lopez A and Karl K: Ratio of fetal choroid plexus to head size: Simple sonographic marker of open spina bifida at 11-13 weeks' gestation. Ultrasound Obstet Gynecol 55: 81-86, 2020.

4. Ushakov F, Sacco A, Andreeva E, Tudorache S, Everett T, David AL and Pandya PP: Crash sign: New first-trimester sonographic marker of spina bifida. Ultrasound Obstet Gynecol 54: 740-745, 2019

5. Lachmann R, Chaoui R, Moratalla J, Picciarelli G and Nicolaides KH: Posterior brain in fetuses with open spina bifida at 11 to 13 weeks. Prenat Diagn 31: 103-106, 2011.

6. Chaoui R, Benoit B, Mitkowska-Wozniak H, Heling KS and Nicolaides KH: Assessment of intracranial translucency (IT) in the detection of spina bifida at the 11-13-week scan. Ultrasound Obstet Gynecol 34: 249-252, 2009.

7. Chaoui R and Nicolaides KH: Detecting open spina bifida at the 11-13-week scan by assessing intracranial translucency and the posterior brain region: Mid-sagittal or axial plane? Ultrasound Obstet Gynecol 38: 609-612, 2011.

8. Lachmann R, Picciarelli G, Moratalla J, Greene N and Nicolaides KH: Frontomaxillary facial angle in fetuses with spina bifida at 11-13 weeks' gestation. Ultrasound Obstet Gynecol 36 268-271, 2010

9. Chen FC, Gerhardt J, Entezami M, Chaoui R and Henrich W: Detection of spina bifida by first trimester screening - Results of the prospective multicenter Berlin IT-Study. Ultraschall Med 38: 151-157, 2017.

10. Ramkrishna J, Araujo Júnior E, Peixoto AB, Da Silva Costa F and Meagher S: Maxillo-occipital line: A sonographic marker for screening of open spina bifida in the first trimester of pregnancy. J Matern Fetal Neonatal Med 32: 4073-4079, 2019.

11. Karl K, Benoit B, Entezami M, Heling KS and Chaoui R: Small biparietal diameter in fetuses with spina bifida on 11-13-week and mid-gestation ultrasound. Ultrasound Obstet Gynecol 40: $140-144,2012$
12. Finn M, Sutton D, Atkinson S, Ransome K, Sujenthiran $P$, Ditcham V, Wakefield P and Meagher S: The aqueduct of Sylvius: A sonographic landmark for neural tube defects in the first trimester. Ultrasound Obstet Gynecol 38: 640-645, 2011.

13. Wertaschnigg D, Ramkrishna J, Ganesan S, Tse C, Scheier M, Volpe N, Ghi T, Meagher S and Rolnik DL: Cranial sonographic markers of fetal open spina bifida at 11 to 13 weeks of gestation. Prenat Diagn 40: 365-372, 2020.

14. Verburg BO, Steegers EA, De Ridder M, Snijders RJ, Smith E, Hofman A, Moll HA, Jaddoe VW and Witteman JC: New charts for ultrasound dating of pregnancy and assessment of fetal growth: Longitudinal data from a population-based cohort study. Ultrasound Obstet Gynecol 31: 388-396, 2008

15. Salomon LJ, Alfirevic Z, Bilardo CM, Chalouhi GE, Ghi T, Kagan KO, Lau TK, Papageorghiou AT, Raine-Fenning NJ, Stirnemann $\mathrm{J}$, et al: ISUOG practice guidelines: Performance of first-trimester fetal ultrasound scan. Ultrasound Obstet Gynecol 41: 102-113, 2013.

16. Kappou D, Papastefanou I, Pilalis A, Kavalakis I, Kassanos D and Souka AP: Towards detecting open spina bifida in the first trimester: The examination of the posterior brain. Fetal Diagn Ther 37: 294-300, 2015.

17. Buisson O, De Keersmaecker B, Senat MV, Bernard JP, Moscoso G and Ville Y: Sonographic diagnosis of spina bifida at 12 weeks: Heading towards indirect signs. Ultrasound Obstet Gynecol 19: 290-292, 2002.

18. Papastefanou I, Souka AP, Pilalis A, Panagopoulos P and Kassanos D: Fetal intracranial translucency and cisterna magna at 11 to 14 weeks: Reference ranges and correlation with chromosomal abnormalities. Prenat Diagn 31: 1189-1192, 2011.

19. Scheier M, Lachmann R, Pětroš $M$ and Nicolaides KH: Three-dimensional sonography of the posterior fossa in fetuses with open spina bifida at 11-13 weeks' gestation. Ultrasound Obstet Gynecol 38: 625-629, 2011.

20. Sacco A, Simpson L, Deprest J and David AL: A study to assess global availability of fetal surgery for myelomeningocele. Prenat Diagn 38: 1020-1027, 2018.

This work is licensed under a Creative Commons Attribution-NonCommercial-NoDerivatives 4.0 International (CC BY-NC-ND 4.0) License. 